Article

\title{
Long-Range Correlations between Observables in a Model with Translational Invariance in Rapidity
}

\author{
Svetlana Belokurova and Vladimir Vechernin * \\ Department of High Energy Physics and Elementary Particles, Saint-Petersburg State University, \\ 7-9 Universitetskaya emb., 199034 St. Petersburg, Russia; sveta.1596@mail.ru \\ * Correspondence: v.vechernin@spbu.ru
}

Received: 30 May 2020; Accepted: 24 June 2020; Published: 2 July 2020

\begin{abstract}
We estimate the impact of the fixation of the total number of sources (quark-gluon strings) on the long-range rapidity correlations between different observables. In our approach this condition models the fixation of the collision centrality class, what is the usual practice in modern collider experiments, like Relativistic Heavy Ion Collider (RHIC), Large Hadron Collider (LHC) and so on. The estimates are obtained under the assumption of the translational invariance in rapidity, which is usually assumed in mid-rapidity region at high energies. Based on these assumptions, we are developing a technique for the analytical calculation of various average values of extensive and intense variables at high string densities on the transverse lattice, taking into account the effects of string fusion, leading to the formation of string clusters. Using this technique we calculate the asymptotes of the correlations coefficients both between the multiplicities and between the multiplicity and the event-mean transverse momentum of particles in two separated rapidity intervals. As a result, we found that fixing the total number of strings has a significant effect on the behavior of both types of correlations, especially in the case of a uniform distribution of strings in the transverse plane.
\end{abstract}

Keywords: strong interaction; high energy; multiparticle production; multiplicity; transverse momentum; forward-backward correlations; long-range rapidity correlations; translation invariance in rapidity; quark-gluon strings; string fusion

\section{Introduction}

In modern particle physics, one of the urgent tasks is to extract information about the initial stage of high-energy hadronic interactions. The valuable source of such information can be the experimental and theoretical studies of the long-range correlations (LRCs) between observables in two separated rapidity windows usually refereed as forward (F) and backward (B) [1]. In this approach one suggests that at the initial stage of the strong interaction at high energy the formation of boost invariant flux tubes of color fields take place between colliding hadrons. It is important that the long-range rapidity correlations originating to the formation of the color flux tubes persist during the evolution of the Quark Gluon Plasma formed later in the collision and hence can be observed experimentally as the LRC between produced particles.

In the framework of the similar approach, rather long ago, in paper [2] the study of the long-range forward-backward correlations between multiplicities $(n)$ in two separated rapidity intervals has been proposed with the aim to find signatures of the string fusion and percolation phenomenon [3-6] in ultrarelativistic heavy ion collisions. It was found later that the investigations of the FB correlations 
involving along with extensive, $n$, also intense observables e.g., such as the event-mean transverse momentum [7-18],

$$
p_{t}=\frac{1}{n} \sum_{i=1}^{n}\left|\mathbf{p}_{t}^{i}\right|
$$

or going to more sophisticated correlation variables, e.g., to the so-called strongly intensive quantities [19-23], enable us to obtain a clearer signal about the initial stage of hadronic interaction, including the process of string fusion, compared to usual FB multiplicity correlations. In the present paper we estimate the impact of the fixing of the total number of sources (quark-gluon strings) on the value of LRC both between the multiplicities in the forward $\left(n_{F}\right)$ and backward $\left(n_{B}\right)$ rapidity windows and between the multiplicity $\left(n_{F}\right)$ in the forward window and the event-mean transverse momentum $\left(p_{t B}\right)$ in the backward one. In our approach the fixing of the total number of strings models the fixation of the event centrality class, what is currently a widespread practice in analyzing experimental data in modern collider experiments (Relativistic Heavy Ion Collider-RHIC, Large Hadron Collider-LHC and so on).

The estimates are obtained under the assumption of validity of the translational (boost) invariance in rapidity, which is usually assumed in the central rapidity region for symmetric reactions at high energies. This assumption implies the uniform rapidity distribution of multiplicity and the dependence of the two-particle correlation function $C_{2}\left(y_{1}, y_{2}\right)$ only on the difference $\Delta y=y_{1}-y_{2}$ of the particle rapidities $y_{1}$ and $y_{2}$ [24].

For symmetric reactions the uniformity of the rapidity distribution of multiplicity is approximately fulfilled at $|y|<1$ and $|y|<2$ for the RHIC and LHC energies correspondingly (see e.g., [25-27]). Referring to the ALICE data, $[26,27])$, one must to take into account that in these papers the distributions are presented in pseudorapidity not in rapidity, what leads to the characteristic kinematical drop of the spectra in the vicinity of $y=0$, which is absent in the rapidity distributions. The dependence of the two-particle correlation function $C_{2}\left(y_{1}, y_{2}\right)$ only on the difference $\Delta y=y_{1}-y_{2}$ of the particle rapidities in the central region is also commonly used when extracting this correlation function from experimental data both at RHIC and LHC energies (see e.g., [28-30]).

Note that for asymmetric reactions, like the proton-nucleus and deuteron-nucleus interactions, the boost invariance in rapidity is absent even in the central region. The rapidity distribution of multiplicity is not uniform at mid-rapidities [31,32] and basically one must to take into account the dependence of the two-particle correlation function $C_{2}\left(y_{1}, y_{2}\right)$ both on $y_{1}$ and $y_{2}$ in this case (see e.g., discussion in paper [33]).

Calculations of the asymptotes of the correlation coefficients are carried out by introducing a lattice (grid) in the impact parameter plane, which enables effectively to take into account the influence of the color string fusion processes, leading to the formation of string clusters in ultra relativistic heavy ion collisions. We present in details the developed methods for the analytical calculation of various average values of extensive and intensive variables at high string densities on the transverse lattice, what was announced in our short note [34], published as the proceedings of the WPCF Conference.

Basing on the averages found with high accuracy we calculate the strength of the LRC between the multiplicities $\left(n_{B}-n_{F}\right)$ and between the multiplicity and the event-mean transverse momentum $\left(p_{t B}-n_{F}\right)$ in the FB observation windows. It turns out that the fixation of the total number of strings, has a significant impact on the behavior of the both type of the correlations.

The paper organized as follows. In Section 2 we introduce the definitions of the $n-n$ and $p_{t}-n$ FB correlation coefficients and briefly describe our model with a lattice in transverse plane, which enables to take into account the string fusion effects on the correlation strength. Section 3 presents the developed method for the analytical calculation of various averages at high string densities on a transverse lattice. In Section 4 basing on the calculated averages we found the covariances $\operatorname{cov}\left(n_{B}, n_{F}\right)$ and $\operatorname{cov}\left(p_{t B}, n_{F}\right)$ determining the LRC coefficients, $b_{n n}$ and $b_{p_{t} n}$, for the cases of uniform and non-uniform distribution of strings in the transverse plane. In Section 5 we summarize the influence of the fixation of the total number of strings (imitating in our approach the fixation of the 
collision centrality) on the behavior of the asymptotes of the LRC $n-n$ and $p_{t}-n$ coefficients at high string density.

\section{The Model with the String Fusion on a Transverse Lattice}

To quantify the strength of the FB correlations between observables measured in two separated rapidity intervals $\delta y_{F}$ and $\delta y_{B}$ it is convenient to use the correlation coefficients defined between the so-called relative variables $n /\langle n\rangle$ and $p_{t} /\left\langle p_{t}\right\rangle$ (see [24,35]). Therefore for the $n-n$ correlation between multiplicities $n_{F}$ and $n_{B}$ in forward and backward rapidity intervals we will use the following definition:

$$
b_{n n} \equiv \frac{\left\langle n_{F}\right\rangle}{\left\langle n_{B}\right\rangle} \frac{\operatorname{cov}\left(n_{B}, n_{F}\right)}{D_{n_{F}}}=\frac{\left\langle n_{F}\right\rangle}{\left\langle n_{B}\right\rangle} \frac{\left\langle n_{B} n_{F}\right\rangle-\left\langle n_{B}\right\rangle\left\langle n_{F}\right\rangle}{\left\langle n_{F}^{2}\right\rangle-\left\langle n_{F}\right\rangle^{2}}
$$

where $D_{n_{F}}$ is the variance of the $n_{F}$. Correspondingly, for the $p_{t}-n$ correlation between the multiplicity, $n_{F}$, in the forward window and the event-mean transverse momentum of the particles observed and in the backward window, $p_{t B}$, we will use the similar definition:

$$
b_{p_{t} n} \equiv \frac{\left\langle n_{F}\right\rangle}{\left\langle p_{t B}\right\rangle} \frac{\operatorname{cov}\left(p_{t B}, n_{F}\right)}{D_{n_{F}}}=\frac{\left\langle n_{F}\right\rangle}{\left\langle p_{t B}\right\rangle} \frac{\left\langle p_{t B} n_{F}\right\rangle-\left\langle p_{t B}\right\rangle\left\langle n_{F}\right\rangle}{\left\langle n_{F}^{2}\right\rangle-\left\langle n_{F}\right\rangle^{2}} .
$$

One can find the value of these correlations considering the effects from the interaction between strings in the framework of the models with string fusion and percolation [3-6]. In the present paper we will take these effects into account in simplified form, by introducing the finite lattice (the grid) in the transverse plane of the collision. This approach was suggested in paper [8]. Later, it was used for a description of various phenomena in ultra relativistic nuclear collisions (azimuthal flows, correlations, the ridge) [9-18,36-40].

In this approach we split the transverse plane into $M$ cells. The area of each cell is equal to the transverse area of a single string. Then we consider that all initial strings, which centers occur in a given cell, merge into one string cluster. In this simplified model each string configuration is completely specified by the set of integers: $C_{\eta}=\left\{\eta_{1}, \ldots, \eta_{M}\right\}$, where $\eta_{i}$ is a number of initial strings merged in a given $i$-th cell.

In fact, in this approach, the transverse plane is divided into cells with different, fluctuating values of the color field inside them. That is similar to the considering the color field density variation in the impact parameter plane in models based on the Color Glass Condensate (CGC) approach [1,41].

So we will suppose that in each cell the $\eta_{i}$ fluctuates around some average, $\bar{\eta}_{i}$, with a scaled variance $\omega$. Physically the $\bar{\eta}_{i}$ are determined by the geometry of a hadronic collision at given value of the impact parameter. Then in accordance with the string fusion prescriptions $[5,6]$ the mean number of charged particles in given observation rapidity window $\delta y$, produced from the fragmentation of a string cluster in the $i$-th cell, and their mean transverse momentum are given by the expressions:

$$
\bar{n}_{i}=\mu \sqrt{\eta_{i}}, \quad \bar{p}_{t}^{i}=p_{0} \sqrt[4]{\eta_{i}},
$$

where $\mu$ and $p_{0}$ is the average multiplicity and the average transverse momentum for particles formed in the decay of a single string.

Note that we assume the translational invariance of the string picture in rapidity, originating from the locality of the strong interaction in the rapidity space, which is usually assumed in the central rapidity region at LHC energies. This translational invariance in rapidity corresponds to the boost invariance of the flux tubes in CGC models [1]. It leads to the independence of the $\bar{n}_{i}$ and $\bar{p}_{t}^{i}$ on a rapidity for a given string configuration $C_{\eta}$ in our model.

We will also assume independent fragmentation of each string cluster into acceptances of the forward and backward windows, because in the present work we are only interested in studying 
long-range correlations, i.e., we will suppose that the $n_{i}^{F}$ and $n_{i}^{B}$ fluctuates independently around their mean values with some scaled variance $\omega_{\mu}$. One can find a more detailed description of the model in $[8,14,16]$.

In the present paper for simplicity we will restrict ourselves to the case of a symmetric reaction and symmetric windows, $\delta y_{F}=\delta y_{B} \equiv \delta y$. For this case in definitions (2) and (3) of the FB correlation coefficients we have $\mu_{F}=\mu_{B} \equiv \mu,\left\langle n_{F}\right\rangle=\left\langle n_{B}\right\rangle \equiv\langle n\rangle, D_{n_{F}}=D_{n_{B}} \equiv D_{n},\left\langle p_{t F}\right\rangle=\left\langle p_{t B}\right\rangle \equiv\left\langle p_{t}\right\rangle$, and so on. Due to mentioned translation invariance in rapidity these quantities do not depend on rapidity. In this case one can also show, [16], that for the LRC:

$$
\left\langle n_{F}^{2}\right\rangle=\left\langle n_{B}^{2}\right\rangle=\left\langle n^{2}\right\rangle=\sum_{i=1}^{M} d_{n_{i}}+\left\langle n_{F} n_{B}\right\rangle=\omega_{\mu}\langle n\rangle+\left\langle n_{F} n_{B}\right\rangle
$$

In the last transition we have used the assumption that the variance $d_{n_{i}} \equiv\left\langle n_{i}^{2}\right\rangle-\left\langle n_{i}\right\rangle^{2}$ of the number of particles, $n_{i}$, produced in any rapidity window from string cluster decay in $i$-th lattice cell, is proportional to their mean multiplicity, $d_{n_{i}}=\omega_{\mu}\left\langle n_{i}\right\rangle$, with the same factor $\omega_{\mu}$. Then

$$
D_{n_{F}}=D_{n_{B}}=D_{n}=\left\langle n^{2}\right\rangle-\langle n\rangle^{2}=\omega_{\mu}\langle n\rangle+\operatorname{cov}\left(n_{F}, n_{B}\right) .
$$

Hence we can find the LRC coefficients using the formula

$$
b_{n n}=\frac{\operatorname{cov}\left(n_{B}, n_{F}\right)}{\omega_{\mu}\langle n\rangle+\operatorname{cov}\left(n_{B}, n_{F}\right)}, \quad b_{p_{t} n}=\frac{\langle n\rangle}{\left\langle p_{t}\right\rangle} \frac{\operatorname{cov}\left(p_{t B}, n_{F}\right)}{\omega_{\mu}\langle n\rangle+\operatorname{cov}\left(n_{B}, n_{F}\right)} .
$$

instead of the general definitions (2) and (3). So to find these correlation coefficients we need to calculate only the following averages: $\langle n\rangle,\left\langle p_{t}\right\rangle,\left\langle n_{B} n_{F}\right\rangle$ and $\left\langle p_{t B} n_{F}\right\rangle$.

As was shown in $[8,9,11,12,14,16]$ in this model with Gaussian distributions one can find the asymptotes of the long-range FB correlation coefficients at large string density in an explicit analytical form. In all these papers we supposed that the number of strings, $\eta_{i}$, in each cell fluctuates around their mean values $\bar{\eta}_{i}$ independently. In the present work we impose the additional condition fixing the total number of initial strings, $N$, in each event and study its impact on the LRC coefficients in the asymptotic regime of high string density.

For this purpose in present analysis we use the following event-by-event string distribution:

$$
P\left(\eta_{1}, \ldots, \eta_{M}\right)=\sqrt{2 \pi \omega \bar{N}} \delta(N-\bar{N}) \prod_{i=1}^{M} \frac{1}{\sqrt{2 \pi \omega \bar{\eta}_{i}}} e^{-\frac{\left(\eta_{i}-\bar{\eta}_{i}\right)^{2}}{2 \omega \bar{\eta}_{i}}}
$$

where $N=\sum_{i=1}^{M} \eta_{i}$ and $\bar{N}=\sum_{i=1}^{M} \bar{\eta}_{i}$. At high string density we can consider $\eta_{i}$, as continuous variables $[8,14,16]$. So the distribution (8) is normalized as follows

$$
\int P\left(\eta_{1}, \ldots, \eta_{M}\right) d \eta_{1} \ldots d \eta_{M}=1 .
$$

Below we will denote by $\langle\ldots\rangle\rangle$ the averages over string configurations, calculated with this distribution. One can easy check that the mean number of strings in each cell $\left\langle\eta_{i}\right\rangle$ is equal to the parameter $\bar{\eta}_{i},\left\langle\eta_{i}\right\rangle=\bar{\eta}_{i}$. In the following consideration the important role will play the variables

$$
v_{i}=\eta_{i}-\bar{\eta}_{i}
$$

characterizing the deviation of $\eta_{i}$ from $\bar{\eta}_{i}$. One can easily verify that for the string distribution (8) we have the following exact relations:

$$
\langle 1\rangle=1, \quad\left\langle v_{k}\right\rangle=0, \quad\left\langle v_{k}^{2}\right\rangle=\bar{\eta}_{k} \omega\left(1-\frac{\bar{\eta}_{k}}{\bar{N}}\right), \quad\left\langle v_{k}^{4}\right\rangle=3 \bar{\eta}_{k}^{2} \omega^{2}\left(1-\frac{\bar{\eta}_{k}}{\bar{N}}\right)^{2},
$$




$$
\begin{gathered}
\left\langle v_{k} v_{m}\right\rangle=-\bar{\eta}_{k} \bar{\eta}_{m} \frac{\omega}{\bar{N}}, \quad\left\langle v_{k} v_{m}^{3}\right\rangle=-3 \bar{\eta}_{k} \bar{\eta}_{m}^{2} \frac{\omega^{2}}{\bar{N}}\left(1-\frac{\bar{\eta}_{m}}{\bar{N}}\right), \quad\left\langle v_{i} v_{k} v_{m}^{2}\right\rangle=-\bar{\eta}_{i} \bar{\eta}_{k} \bar{\eta}_{m} \frac{\omega^{2}}{\bar{N}}\left(1-3 \frac{\bar{\eta}_{m}}{\bar{N}}\right), \\
\left\langle v_{k}^{2} v_{m}^{2}\right\rangle=\bar{\eta}_{k} \bar{\eta}_{m} \omega^{2}\left(1-\frac{\bar{\eta}_{k}}{\bar{N}}-\frac{\bar{\eta}_{m}}{\bar{N}}+3 \frac{\bar{\eta}_{k} \bar{\eta}_{m}}{\bar{N}^{2}}\right), \quad\left\langle v_{i} v_{j} v_{k} v_{m}\right\rangle=3 \bar{\eta}_{i} \bar{\eta}_{j} \bar{\eta}_{k} \bar{\eta}_{m} \frac{\omega^{2}}{\bar{N}^{2}} .
\end{gathered}
$$

If $\alpha \equiv \sum_{i=1}^{n} \alpha_{i}$ is odd, then we have

$$
\left\langle v_{i}^{\alpha_{1}} v_{j}^{\alpha_{2}} v_{k}^{\left.\alpha_{3} \ldots\right\rangle}\right\rangle=0, \quad \text { at } \quad \alpha \equiv \sum_{i=1}^{n} \alpha_{i}=2 l+1
$$

We note that the relations (10) are valid only if $i \neq j \neq k \neq m$. Really, we see that $\left.\left\langle v_{k} v_{m}\right\rangle\right|_{k=m} \neq\left\langle v_{k}^{2}\right\rangle$, $\left.\left\langle v_{k} v_{m}^{3}\right\rangle\right|_{k=m} \neq\left\langle v_{k}^{4}\right\rangle$ and so on. That is a consequence of the correlations between fluctuations of the $\eta_{i}$ in different cells arising due to the conservation of the total number of strings (see [34]).

Use of the relations (10) and (11) enables drastically simplify the calculation of various averages in this model, because all integrations over the $\eta_{i}$ come down to using these simple rules.

We will calculate the asymptotes of the LRC coefficients (7) at high string density supposing that all $\bar{\eta}_{i} \gg 1$. We will also suppose that $M \gg 1$, because as it was discussed in $[15,16]$ with a realistic string radius $r_{s t r}=0.2 \div 0.3 \mathrm{fm}$ we need lattices with a large number of cells $M \sim 10^{2}$ and $10^{4}$ for a description of pp and AA collisions correspondingly.

\section{Averaging over String Configurations}

We will demonstrate the technique of the analytical calculation of the different lattice averages with the distribution (8) by using as example the most complicated calculation of the mean value of the intensive variable - the mean transverse momentum of the produced particles, $\left\langle p_{t}\right\rangle$.

Regarding the accuracy of the calculation, we need to take into account the terms of the order $1 / \bar{\eta}, 1 / \bar{\eta}^{2}, 1 /(M \bar{\eta})$ and $1 /\left(M \bar{\eta}^{2}\right)$. Because, as we will see later, the terms of the leading order in $M$ (the $1 / \bar{\eta}$ and $1 / \bar{\eta}^{2}$ in the case of $\left\langle p_{t}\right\rangle$ calculation) are cancelled when calculating the covariances entering expressions (7) for the LRC coefficients $b_{n n}$ and $b_{p_{t} n}$. Moreover, in the case of homogeneous string spreading in transverse plane, when all $\bar{\eta}_{i}=\bar{\eta}$, we have additional mutual cancellation of the contributions of the order of $1 /(M \bar{\eta})$ to the LRC coefficients calculated with the distribution (8) corresponding to a fixed total number of initial strings. In last case the only contribution to the LRC coefficient originates from the terms of the order of $1 /\left(M \bar{\eta}^{2}\right)$.

As was shown in [16] with the prescriptions (4) we can find $\left\langle p_{t}\right\rangle$ by calculating the following average over string configurations:

$$
\frac{\left\langle p_{t B}\right\rangle}{p_{0}}=\left\langle\frac{\sum_{i=1}^{M} \eta_{i}^{\frac{3}{4}}}{\sum_{k=1}^{M} \eta_{i}^{\frac{1}{2}}}\right\rangle \equiv\langle Y Z\rangle
$$

Here we introduce the following notations

$$
Y \equiv \sum_{i=1}^{M} \eta_{i}^{\frac{3}{4}}, \quad Z \equiv\left(\sum_{i=1}^{M} \eta_{i}^{\frac{1}{2}}\right)^{-1} .
$$

Taking into account the definition (9) we can present the $Y$ with the accuracy up to $v_{i}^{4}$ as follows

$$
Y=M S_{3 / 4}\left[1+\frac{1}{M S_{3 / 4}} \sum_{i=1}^{M} \bar{\eta}_{i}^{\frac{3}{4}}\left(\frac{3 v_{i}}{4 \bar{\eta}_{i}}-\frac{3 v_{i}^{2}}{32 \bar{\eta}_{i}^{2}}+\frac{15 v_{i}^{3}}{384 \bar{\eta}_{i}^{3}}-\frac{45 v_{i}^{4}}{2048 \bar{\eta}_{i}^{4}}\right)\right],
$$


where we have introduced the following convenient notation

$$
S_{\beta} \equiv \frac{1}{M} \sum_{i=1}^{M} \bar{\eta}_{i}^{\beta} .
$$

To calculate the $Z$ with the same accuracy we at first have to use the expansion

$$
\sum_{i=1}^{M} \eta_{i}^{\frac{1}{2}}=M S_{1 / 2}\left[1+\frac{1}{M S_{1 / 2}} \sum_{i=1}^{M} \bar{\eta}_{i}^{\frac{1}{2}}\left(\frac{v_{i}}{2 \bar{\eta}_{i}}-\frac{v_{i}^{2}}{8 \bar{\eta}_{i}^{2}}+\frac{v_{i}^{3}}{16 \bar{\eta}_{i}^{3}}-\frac{5 v_{i}^{4}}{128 \bar{\eta}_{i}^{4}}\right)\right] \equiv M S_{1 / 2}[1+a],
$$

where

$$
a=\frac{1}{M S_{1 / 2}} \sum_{i=1}^{M} \bar{\eta}_{i}^{\frac{1}{2}}\left(\frac{v_{i}}{2 \bar{\eta}_{i}}-\frac{v_{i}^{2}}{8 \bar{\eta}_{i}^{2}}+\frac{v_{i}^{3}}{16 \bar{\eta}_{i}^{3}}-\frac{5 v_{i}^{4}}{128 \bar{\eta}_{i}^{4}}\right) .
$$

Then we can write

$$
Z \equiv \frac{1}{M S_{1 / 2}[1+a]}=\frac{1}{M S_{1 / 2}}\left[1-a+a^{2}-a^{3}+a^{4}\right]
$$

Multiplying $Y$ by $Z$ and taking into account only the terms $v_{i}^{\alpha_{1}} v_{j}^{\alpha_{2}} v_{k}^{\alpha_{3}} v_{m}^{\alpha_{4}}$, satisfying the conditions $\alpha=\sum_{i} \alpha_{i}=2$ or 4 (see Formulas (10) and (11)), we find

$$
Y Z=\frac{S_{3 / 4}}{S_{1 / 2}}\left[1+A_{1}+A_{2}+B_{1}+B_{2}+C\right] .
$$

Here the $A_{1}$ and $A_{2}$ collects four terms with $\alpha=2$ :

$$
\begin{gathered}
A_{1}=\frac{1}{8 M S_{1 / 2}} \sum_{i=1}^{M} \frac{v_{i}^{2}}{\bar{\eta}_{i}^{3 / 2}}-\frac{3}{32 M S_{3 / 4}} \sum_{i=1}^{M} \frac{v_{i}^{2}}{\bar{\eta}_{i}^{5 / 4}}, \\
A_{2}=\frac{1}{4 M^{2} S_{1 / 2}^{2}} \sum_{i, j}^{M} \frac{v_{i} v_{j}}{\bar{\eta}_{i}^{1 / 2} \bar{\eta}_{j}^{1 / 2}}-\frac{3}{8 M^{2} S_{1 / 2} S_{3 / 4}} \sum_{i, j}^{M} \frac{v_{i} v_{j}}{\bar{\eta}_{i}^{1 / 2} \bar{\eta}_{j}^{1 / 4}}
\end{gathered}
$$

and the $B_{1}, B_{2}$ and the $C$ collect 12 terms with $\alpha=4$ :

$$
\begin{gathered}
B_{1}=\frac{5}{128 M S_{1 / 2}} \sum_{i=1}^{M} \frac{v_{i}^{4}}{\bar{\eta}_{i}^{7 / 2}}-\frac{45}{2048 M S_{3 / 4}} \sum_{i=1}^{M} \frac{v_{i}^{4}}{\bar{\eta}_{i}^{13 / 4}} \\
+\frac{1}{64 M^{2} S_{1 / 2}^{2}} \sum_{i, j}^{M} \frac{v_{i}^{2} v_{j}^{2}}{\bar{\eta}_{i}^{3 / 2} \bar{\eta}_{j}^{3 / 2}}-\frac{3}{256 M^{2} S_{1 / 2} S_{3 / 4}} \sum_{i, j}^{M} \frac{v_{i}^{2} v_{j}^{2}}{\bar{\eta}_{i}^{3 / 2} \bar{\eta}_{j}^{5 / 4}} \\
B_{2}=\frac{1}{16 M^{2} S_{1 / 2}^{2}} \sum_{i, j}^{M} \frac{v_{i} v_{j}^{3}}{\bar{\eta}_{i}^{1 / 2} \bar{\eta}_{j}^{5 / 2}}-\frac{5}{256 M^{2} S_{1 / 2} S_{3 / 4}} \sum_{i, j}^{M} \frac{v_{i} v_{j}^{3}}{\bar{\eta}_{i}^{1 / 2} \bar{\eta}_{j}^{9 / 4}}-\frac{3}{64 M^{2} S_{1 / 2} S_{3 / 4}} \sum_{i, j}^{M} \frac{v_{i} v_{j}^{3}}{\bar{\eta}_{i}^{1 / 4} \bar{\eta}_{j}^{5 / 2}} \\
-\frac{3}{32 M^{3} S_{1 / 2}^{3}} \sum_{i, j, k}^{M} \frac{v_{i} v_{j} v_{k}^{2}}{\bar{\eta}_{i}^{1 / 2} \bar{\eta}_{j}^{1 / 2} \bar{\eta}_{k}^{3 / 2}}-\frac{3}{128 M^{3} S_{1 / 2}^{2} S_{3 / 4}} \sum_{i, j, k}^{M} \frac{v_{i} v_{j} v_{k}^{2}}{\bar{\eta}_{i}^{1 / 2} \bar{\eta}_{j}^{1 / 2} \bar{\eta}_{k}^{5 / 4}}-\frac{3}{32 M^{3} S_{1 / 2}^{2} S_{3 / 4}} \sum_{i, j, k}^{M} \frac{v_{i} v_{j} v_{k}^{2}}{\bar{\eta}_{i}^{1 / 2} \bar{\eta}_{j}^{1 / 4} \bar{\eta}_{k}^{3 / 2}}, \\
C=\frac{1}{16 M^{4} S_{1 / 2}^{4}} \sum_{i, j, k, m}^{M} \frac{v_{i} v_{j} v_{k} v_{m}}{\bar{\eta}_{i}^{1 / 2} \bar{\eta}_{j}^{1 / 2} \bar{\eta}_{k}^{1 / 2} \bar{\eta}_{m}^{1 / 2}}-\frac{3}{32 M^{4} S_{1 / 2}^{3} S_{3 / 4}} \sum_{i, j, k, m}^{M} \frac{v_{i} v_{j} v_{k} v_{m}}{\bar{\eta}_{i}^{1 / 2} \bar{\eta}_{j}^{1 / 2} \bar{\eta}_{k}^{1 / 2} \bar{\eta}_{m}^{1 / 4}}
\end{gathered}
$$


We will see below that the leading contributions to $\left\langle p_{t}\right\rangle$ originating from the terms $A_{1}, A_{2}, B_{1}, B_{2}$ and $C$ are of the following order:

$$
A_{1} \sim \frac{1}{\bar{\eta}}, \quad A_{2} \sim \frac{1}{M \bar{\eta}}, \quad B_{1} \sim \frac{1}{\bar{\eta}^{2}}, \quad B_{2} \sim \frac{1}{M \bar{\eta}^{2}}, \quad C \sim \frac{1}{M^{2} \bar{\eta}^{2}} .
$$

So, taking into account the remark in the beginning of the present Section in the leading approximation we can do not take the $C$ contribution into consideration.

Now to calculate the $\left\langle p_{t}\right\rangle$ by (12) we need to average the expression (19) over string fluctuations, given by the distribution (8):

$$
\frac{\left\langle p_{t}\right\rangle}{p_{0}}=\langle Y Z\rangle=\frac{S_{3 / 4}}{S_{1 / 2}}\left[1+\left\langle A_{1}\right\rangle+\left\langle A_{2}\right\rangle+\left\langle B_{1}\right\rangle+\left\langle B_{2}\right\rangle+\langle C\rangle\right] .
$$

We can do this using the rules (10) and (11) obtained above. At that we have to take into account that these rules are valid only for non coinciding arguments (see the remark after the Formula (11)). So, at first we must express all sums entering the Formulas (20)-(24) through the sums with non coinciding arguments. We can do it easily using the following obvious relations:

$$
\sum_{i, j}=\sum_{i \neq j}+\sum_{i=j}, \quad \sum_{i, j, k}=\sum_{i \neq j \neq k}+\sum_{i=j \neq k}+\sum_{i \neq j=k}+\sum_{i=k \neq j}+\sum_{i=j=k}{ }^{\prime}
$$

and so on. Then for terms of the general form we have

$$
\begin{gathered}
\sum_{i, j} \frac{\left\langle v_{i}^{\alpha_{1}} v_{j}^{\alpha_{2}}\right\rangle}{\bar{\eta}_{i}^{\beta_{1}} \bar{\eta}_{j}^{\beta_{2}}}=\sum_{i \neq j} \frac{\left\langle v_{i}^{\alpha_{1}} v_{j}^{\alpha_{2}}\right\rangle}{\bar{\eta}_{i}^{\beta_{1}} \bar{\eta}_{j}^{\beta_{2}}}+\sum_{i} \frac{\left\langle v_{i}^{\alpha_{1}+\alpha_{2}}\right\rangle}{\bar{\eta}_{i}^{\beta_{1}+\beta_{2}}}, \quad \sum_{i, j, k} \frac{\left\langle v_{i}^{\alpha_{1}} v_{j}^{\alpha_{2}} v_{k}^{\alpha_{3}}\right\rangle}{\bar{\eta}_{i}^{\beta_{1}} \bar{\eta}_{j}^{\beta_{2}} \bar{\eta}_{k}^{\beta_{3}}} \\
=\sum_{i \neq j \neq k} \frac{\left\langle v_{i}^{\alpha_{1}} v_{j}^{\alpha_{2}} v_{k}^{\alpha_{3}}\right\rangle}{\bar{\eta}_{i}^{\beta_{1}} \bar{\eta}_{j}^{\beta_{2}} \bar{\eta}_{k}^{\beta_{3}}}+\sum_{i \neq k} \frac{\left\langle v_{i}^{\alpha_{1}+\alpha_{2}} v_{k}^{\alpha_{3}}\right\rangle}{\bar{\eta}_{i}^{\beta_{1}+\beta_{2}} \bar{\eta}_{k}^{\beta_{3}}}+\sum_{i \neq j} \frac{\left\langle v_{i}^{\alpha_{1}} v_{j}^{\alpha_{2}+\alpha_{3}}\right\rangle}{\bar{\eta}_{i}^{\beta_{1}} \bar{\eta}_{j}^{\beta_{2}+\beta_{3}}}+\sum_{i \neq j} \frac{\left\langle v_{i}^{\alpha_{1}+\alpha_{3}} v_{j}^{\alpha_{2}}\right\rangle}{\bar{\eta}_{i}^{\beta_{1}+\beta_{3}} \bar{\eta}_{j}^{\beta_{2}}}+\sum_{i} \frac{\left\langle v_{i}^{\alpha_{1}+\alpha_{2}+\alpha_{3}}\right\rangle}{\bar{\eta}_{i}^{\beta_{1}+\beta_{2}+\beta_{3}}} .
\end{gathered}
$$

After that, using the rules (10) and (11) and taking also into account that $\bar{N}=M S_{1}$, we find the answer for $\left\langle p_{t}\right\rangle$ as the linear combination of the sums of the following type:

$$
\sum_{i} \bar{\eta}_{i}^{\beta}=M S_{\beta}, \quad \sum_{i \neq j} \bar{\eta}_{i}^{\beta} \bar{\eta}_{j}^{\gamma}, \quad \sum_{i \neq j \neq k} \bar{\eta}_{i}^{\beta} \bar{\eta}_{j}^{\gamma} \bar{\eta}_{k}^{\delta},
$$

and so on. Now to express all these sums through the $S_{\beta}$, defined by (15), we have to use the relations inverse to (27):

$$
\begin{gathered}
\sum_{i \neq j} \bar{\eta}_{i}^{\beta} \bar{\eta}_{j}^{\gamma}=\sum_{i, j} \bar{\eta}_{i}^{\beta} \bar{\eta}_{j}^{\gamma}-\sum_{i=j} \bar{\eta}_{i}^{\beta} \bar{\eta}_{j}^{\gamma}=\left(\sum_{i} \bar{\eta}_{i}^{\beta}\right)\left(\sum_{j} \bar{\eta}_{j}^{\gamma}\right)-\sum_{i} \bar{\eta}_{i}^{\beta+\gamma}=M^{2} S_{\beta} S_{\gamma}-M S_{\beta+\gamma}, \\
\sum_{i \neq j \neq k} \bar{\eta}_{i}^{\beta} \bar{\eta}_{j}^{\gamma} \bar{\eta}_{k}^{\delta}=\sum_{i, j, k} \bar{\eta}_{i}^{\beta} \bar{\eta}_{j}^{\gamma} \bar{\eta}_{k}^{\delta}-\sum_{i=j, k} \bar{\eta}_{i}^{\beta} \bar{\eta}_{j}^{\gamma} \bar{\eta}_{k}^{\delta}-\sum_{i, j=k} \bar{\eta}_{i}^{\beta} \bar{\eta}_{j}^{\gamma} \bar{\eta}_{k}^{\delta}-\sum_{i=k, j} \bar{\eta}_{i}^{\beta} \bar{\eta}_{j}^{\gamma} \bar{\eta}_{k}^{\delta}+2 \sum_{i=j=k} \bar{\eta}_{i}^{\beta} \bar{\eta}_{j}^{\gamma} \bar{\eta}_{k}^{\delta} \\
=M^{3} S_{\beta} S_{\gamma} S_{\delta}-M^{2} S_{\beta} S_{\gamma+\delta}-M^{2} S_{\gamma} S_{\beta+\delta}-M^{2} S_{\delta} S_{\beta+\gamma}+2 M S_{\beta+\gamma+\delta} .
\end{gathered}
$$

Using this technique we can easily check that the leading orders of the terms in the $A_{1}, A_{2}, B_{1}, B_{2}$ and the $C$ are given by (25). Then, applying this approach and taking into account only the terms of 
the order $1 / \bar{\eta}, 1 / \bar{\eta}^{2}, 1 /(M \bar{\eta})$ and $1 /\left(M \bar{\eta}^{2}\right)$ in the contributions $A_{1}, A_{2}, B_{1}, B_{2}$ (see the remark in the beginning of the present Section) we find

$$
\begin{gathered}
\frac{\left\langle p_{t}\right\rangle}{p_{0}}=\langle Y Z\rangle=\frac{S_{3 / 4}}{S_{1 / 2}}\left\{1+\omega\left(\frac{S_{-1 / 2}}{8 S_{1 / 2}}-\frac{3 S_{-1 / 4}}{32 S_{3 / 4}}\right)+\omega^{2}\left(\frac{S_{-1 / 2}^{2}}{64 S_{1 / 2}^{2}}-\frac{3 S_{-1 / 2} S_{-1 / 4}}{256 S_{1 / 2} S_{3 / 4}}\right.\right. \\
\left.+\frac{15 S_{-3 / 2}}{128 S_{1 / 2}}-\frac{135 S_{-5 / 4}}{2048 S_{3 / 4}}\right)+\frac{1}{M}\left[\omega\left(\frac{3}{32 S_{1}}+\frac{1}{4 S_{1 / 2}^{2}}-\frac{3 S_{1 / 4}}{8 S_{1 / 2} S_{3 / 4}}\right)+\omega^{2}\left(\frac{7 S_{-1}}{32 S_{1 / 2}^{2}}-\frac{3 S_{-1 / 2}}{32 S_{1 / 2}^{3}}\right.\right. \\
\left.\left.\left.-\frac{29 S_{-1 / 2}}{256 S_{1} S_{1 / 2}}-\frac{3 S_{1 / 4} S_{-1 / 2}}{32 S_{1 / 2}^{2} S_{3 / 4}}+\frac{231 S_{-1 / 4}}{1024 S_{1} S_{3 / 4}}-\frac{3 S_{-1 / 4}}{128 S_{1 / 2}^{2} S_{3 / 4}}-\frac{57 S_{-3 / 4}}{256 S_{1 / 2} S_{3 / 4}}\right)\right]\right\}
\end{gathered}
$$

\section{Calculation of the Long-Range Correlation Coefficients}

Using the methods developed in Section 3 with the example of the $\left\langle p_{t}\right\rangle$ calculation, we can now easily find all other averages entering the correlation coefficients $b_{n n}$ and $b_{p_{t} n}$ defined by (7) with necessary accuracy.

In accordance with the prescriptions (4), taking into account (16) and applying the developed technique we find

$$
\frac{\langle n\rangle}{\mu}=\left\langle\sum_{i=1}^{M} \eta_{i}^{\frac{1}{2}}\right\rangle=M S_{1 / 2}\left\{1-\omega \frac{S_{-1 / 2}}{8 S_{1 / 2}}-\omega^{2} \frac{15 S_{-3 / 2}}{128 S_{1 / 2}}+\frac{1}{M}\left[\omega \frac{1}{8 S_{1}}+\omega^{2} \frac{15 S_{-1 / 2}}{64 S_{1} S_{1 / 2}}\right]\right\} .
$$

Using the general expression for $\left\langle p_{t B} n_{F}\right\rangle$ :

$$
\frac{\left\langle p_{t B} n_{F}\right\rangle}{p_{0} \mu}=\left\langle\sum_{i=1}^{M} \eta_{i}^{\frac{3}{4}}\right\rangle
$$

obtained in [16] for the LRC, and the Formula (14) we also find that in the framework of the developed approach:

$$
\frac{\left\langle p_{t B} n_{F}\right\rangle}{p_{0} \mu}=M S_{3 / 4}\left\{1-\omega \frac{3 S_{-1 / 4}}{32 S_{3 / 4}}-\omega^{2} \frac{135 S_{-5 / 4}}{2048 S_{3 / 4}}+\frac{1}{M}\left[\omega \frac{3}{32 S_{1}}+\omega^{2} \frac{135 S_{-1 / 4}}{1024 S_{1} S_{3 / 4}}\right]\right\} .
$$

Finally by this technique taking into account the Formula (16) we find for the contribution of the LRC to $\left\langle n_{B} n_{F}\right\rangle$ the following expression:

$$
\begin{gathered}
\frac{\left\langle n_{B} n_{F}\right\rangle}{\mu^{2}}=\left\langle\sum_{i=1}^{M} \eta_{i}^{\frac{1}{2}} \sum_{j=1}^{M} \eta_{j}^{\frac{1}{2}}\right\rangle=M^{2} S_{1 / 2}^{2}\left\{1-\frac{\omega S_{-1 / 2}}{4 S_{1 / 2}}-\frac{\omega^{2}}{64 S_{1 / 2}}\left(\frac{S_{-1 / 2}^{2}}{S_{1 / 2}}-15 S_{-3 / 2}\right)\right. \\
\left.+\frac{1}{M}\left[\frac{\omega}{4 S_{1 / 2}^{2}}+\frac{\omega^{2}}{4 S_{1 / 2}}\left(\frac{7 S_{-1}}{8 S_{1 / 2}}+\frac{S_{-1 / 2}}{S_{1}}\right)\right]\right\} .
\end{gathered}
$$

Now we can calculate the covariances (the correlators) entering the correlation coefficients $b_{n n}$ and $b_{p_{t} n}$ (see the Formula (7)):

$$
\begin{gathered}
\frac{\operatorname{cov}\left(n_{B}, n_{F}\right)}{\mu^{2}}=\frac{\left\langle n_{B} n_{F}\right\rangle-\langle n\rangle^{2}}{\mu^{2}}=M\left[\frac{\omega}{4}\left(1-\frac{S_{1 / 2}^{2}}{S_{1}}\right)+\frac{\omega^{2}}{32}\left(7 S_{-1}-6 \frac{S_{1 / 2} S_{-1 / 2}}{S_{1}}\right)\right], \\
\frac{\operatorname{cov}\left(p_{t B}, n_{F}\right)}{p_{0} \mu}=\frac{\left\langle p_{t B} n_{F}\right\rangle-\left\langle p_{t}\right\rangle\langle n\rangle}{p_{0} \mu}=\frac{\omega}{4}\left(\frac{3 S_{1 / 4}}{2 S_{1 / 2}}-\frac{S_{3 / 4}}{S_{1 / 2}^{2}}-\frac{S_{3 / 4}}{2 S_{1}}\right)
\end{gathered}
$$




$$
+\frac{\omega^{2}}{8}\left(\frac{57 S_{-3 / 4}}{32 S_{1 / 2}}-\frac{21 S_{-1 / 4}}{32 S_{1}}+\frac{3 S_{-1 / 4}}{16 S_{1 / 2}^{2}}-\frac{7 S_{3 / 4} S_{-1}}{4 S_{1 / 2}^{2}}+\frac{3 S_{1 / 4} S_{-1 / 2}}{8 S_{1 / 2}^{2}}+\frac{S_{3 / 4} S_{-1 / 2}}{S_{1 / 2}^{3}}-\frac{S_{3 / 4} S_{-1 / 2}}{S_{1} S_{1 / 2}}\right) .
$$

We really see that all terms proportional $M^{2}$ in Formula (35) for $\left\langle n_{B} n_{F}\right\rangle$ are cancelled by the terms of this order in $\langle n\rangle^{2}$. similarly, all terms proportional $M$ in Formula (34) for $\left\langle p_{t B} n_{F}\right\rangle$ are cancelled by the terms of this order in the product $\left\langle p_{t}\right\rangle\langle n\rangle$, given by the Formulas (31) and (32).

Moreover if we will go to the case with a homogenous string distribution in the transverse plane with some mean string density, corresponding to the same mean number, $\bar{\eta}$, of initial strings in a lattice (grid) cell, when all $\bar{\eta}_{i}=\bar{\eta}$, then we will have

$$
S_{\beta} \equiv \frac{1}{M} \sum_{i=1}^{M} \bar{\eta}_{i}^{\beta}=\bar{\eta}^{\beta}
$$

In this case all contributions proportional to $\omega$ in Formulas (35) and (34) for the correlators (covariances) $\operatorname{cov}\left(n_{B}, n_{F}\right)$ and $\operatorname{cov}\left(p_{t B}, n_{F}\right)$ are also mutually cancelled and only the contributions of the terms of the order $\omega^{2}$ survive. In this simple case the formula for the correlators (covariances) reduce to

$$
\begin{gathered}
\frac{\operatorname{cov}\left(n_{B}, n_{F}\right)}{\mu^{2}}=M \frac{\omega^{2}}{32 \bar{\eta}}, \\
\frac{\operatorname{cov}\left(p_{t B}, n_{F}\right)}{p_{0} \mu}=-\frac{\omega^{2}}{128 \bar{\eta}^{2}} .
\end{gathered}
$$

This leads to the proportionality of the the $b_{n n}$ and $b_{p_{t} n}$ correlation coefficients (7) at large string density to $1 / \bar{\eta}^{3 / 2}$ in this case, instead of $1 / \sqrt{\bar{\eta}}$ that took place in the case without the fixation of the total string number $[8,9,11,14]$.

At that by (39) and (40) we see that in this case the $b_{p_{t} n}$ correlation coefficient is negative, whereas the $b_{n n}$ correlation coefficient is positive. Note that without imposing this additional condition, fixing the total number of strings, both $b_{n n}$ and $b_{p_{t} n}$ correlation coefficients were always positive for a homogeneous string distribution in the transverse plane $[8,9,11,14]$.

\section{Summary}

We present the developed technique for the analytical calculation of various average values of extensive and intensive variables at high string densities on the transverse lattice with taking into account the string fusion effects leading to the formation of string clusters. Using this technique we calculate the asymptotes of the LRC coefficients between the multiplicities, $b_{n n}$, and between the multiplicity and the event-mean transverse momentum, $b_{p_{t} n}$, in two separated rapidity intervals at high string density and with the fixation of the total number of initial strings. This last condition models in our approach the fixation of the collision centrality class, which is the usual practice of analyzing experimental data in modern collider experiments, like RHIC, LHC and so on.

As a result we found that the fixation of the total number of strings has a significant impact on the behavior of the both type of the correlations, especially in the case of uniform string distribution in transverse plane. In this case at large string density the $b_{n n}$ and $b_{p_{t} n}$ LRC coefficients become proportional to $1 / \bar{\eta}^{3 / 2}$ instead of $1 / \sqrt{\bar{\eta}}$ that took place without the fixation of the total number of strings $[8,9,11,14]$.

We also found that in this case the correlation coefficient $b_{p_{t} n}$ always has a negative value, while the correlation coefficient $b_{n n}$ is positive. Whereas without fixing the total number of strings both correlation coefficients $b_{n n}$ and $b_{p_{t} n}$ were always positive for a homogeneous distribution of the strings in the transverse plane $[8,9,11,14]$.

In general, the proposed lattice approach to the analysis of correlations between various extensive and intense observables can be useful for modeling the magnitude of these correlations under 
developing of various detecting systems aimed to study these effects, in particular, in the design and construction of vertex detectors for the NICA accelerator complex.

Author Contributions: Both authors contributed equally to this work. All authors have read and agreed to the published version of the manuscript.

Funding: This research was funded by the Russian Foundation for Basic Research, project number 18-02-40075.

Acknowledgments: V.V. acknowledges the support given by the Saint-Petersburg State University grant for outgoing academic mobility, id. 41159705.

Conflicts of Interest: The authors declare no conflict of interest.

\title{
Abbreviations
}

The following abbreviations are used in this manuscript:

\author{
LRC Long-Range Correlation \\ FB Forward-Backward \\ CGC Color Glass Condensate \\ RHIC Relativistic Heavy Ion Collider \\ LHC Large Hadron Collider
}

\section{References}

1. Dumitru, A.; Gelis, F.; McLerran, L.; Venugopalan, R. Glasma flux tubes and the near side ridge phenomenon at RHIC. Nucl. Phys. A 2008, 810, 91-108. [CrossRef]

2. Amelin, N.S.; Armesto, N.; Braun, M.A.; Ferreiro, E.G.; Pajares, C. Long and short range correlations and the search of the quark gluon plasma. Phys. Rev. Lett. 1994, 73, 2813. [CrossRef] [PubMed]

3. Biro, T.S.; Nielsen, H.B.; Knoll, J. Colour rope model for extreme relativistic heavy ion collisions. Nucl. Phys. B 1984, 245, 449-468. [CrossRef]

4. Bialas, A.; Czyz, W. Conversion of color field into $q \bar{q}$ matter in the central region of high-energy heavy ion collisions. Nucl. Phys. B 1986, 267, 242-252. [CrossRef]

5. Braun, M.A.; Pajares, C. Particle production in nuclear collisions and string interactions. Phys. Lett. B 1992, 287, 154-158. [CrossRef]

6. Braun, M.A.; Pajares, C. A probabilistic model of interacting strings. Nucl. Phys. B 1993, 390, 542-558. [CrossRef]

7. Braun, M.A.; Pajares, C. Transverse momentum distributions and their forward-backward correlations in the percolating color string approach. Phys. Rev. Lett. 2000, 85, 4864. [CrossRef]

8. Vechernin, V.V.; Kolevatov, R.S. Cellular approach to long-range $p_{T}$ and multiplicity correlations in the string fusion model. Vestn. SPbU Ser. 2004, 4, 11-27.

9. Braun, M.A.; Kolevatov, R.S.; Pajares, C.; Vechernin, V.V. Correlations between multiplicities and average transverse momentum in the percolating color strings approach. Eur. Phys. J. C 2004, 32, 535-546. [CrossRef]

10. Alessandro, B.; Antinori, F.; Belikov, J.A.; Blume, C.; Dainese, A.; Foka, P.; Giubellino, P.; Hippolyte, B.; Kuhn, C.; Martinez, G.; et al. [ALICE Collaboration]. ALICE: Physics Performance Report, Volume II. Phys. J. G 2006, 32, 1295-2040

11. Vechernin, V.V.; Kolevatov, R.S. On multiplicity and transverse-momentum correlations in collisions of ultrarelativistic ions. Phys. Atom. Nucl. 2007, 70, 1797-1808. [CrossRef]

12. Vechernin, V.V.; Kolevatov, R.S. Long-range correlations between transverse momenta of charged particles produced in relativistic nucleus-nucleus collisions. Phys. Atom. Nucl. 2007, 70, 1809-1818. [CrossRef]

13. Kovalenko, V.; Vechernin, V. Long-range rapidity correlations in high energy AA collisions in Monte Carlo model with string fusion. In Proceedings of the XXVth International Nuclear Physics Conference (INPC 2013), Firenze, Italy, 2-7 June 2013; Volume 66, p. 04015.

14. Vechernin, V.V. Correlation Between Transverse Momenta in the String Fusion Model. Theor. Math. Phys. 2015, 184, 1271-1280. [CrossRef] 
15. Vechernin, V. Long-range rapidity correlations between mean transverse momenta in the model with string fusion. In Proceedings of the 19-th International Seminar on High Energy Physics (QUARKS-2016), Pushkin, Russia, 29 May-4 June 2016; Volume 125, p. 04022.

16. Vechernin, V.V. Asymptotic behavior of the correlation coefficients of transverse momenta in the model with string fusion. Theor. Math. Phys. 2017, 190, 251-267. [CrossRef]

17. Belokurova, S. Asymptotes of multiplicity and transverse momentum correlation coefficients at large string density. In Proceedings of the XXth International Seminar on High Energy Physics (QUARKS-2018), Valday, Russia, 27 May-2 June 2018; Volume 191, p. 04010.

18. Belokurova, S.N.; Vechernin, V.V. Strongly intensive variables and the long-range correlations in the model with a lattice in transverse plane. Theor. Math. Phys. 2019, 200, 1094-1109. [CrossRef]

19. Gorenstein, M.I.; Gazdzicki, M. Strongly intensive quantities. Phys. Rev. C 2011, 84, 014904. [CrossRef]

20. Andronov, E.V. Influence of the quark-gluon string fusion mechanism on long-range rapidity correlations and fluctuations. Theor. Math. Phys. 2015, 185, 1383-1390. [CrossRef]

21. Vechernin, V. Short- and long-range rapidity correlations in the model with a lattice in transverse plane. In Proceedings of the XXth International Seminar on High Energy Physics (QUARKS-2018), Valday, Russia, 27 May-2 June 2018; Volume 191, p. 04011.

22. Andronov, E.; Vechernin, V. Strongly intensive observable between multiplicities in two acceptance windows in a string model. Eur. Phys. J. A 2019, 55, 14. [CrossRef]

23. Vechernin, V.; Andronov, E. Strongly intensive observables in the model with string fusion. Universe 2019, 5, 15. [CrossRef]

24. Vechernin, V. Forward-backward correlations between multiplicities in windows separated in azimuth and rapidity. Nucl. Phys. A 2015, 939, 21-45. [CrossRef]

25. Bearden, I.G.; Beavis, D.; Besliu, C.; Budick, B.; Boggild, H.; Chasman, C.; Christensen, C.H.; Christiansen, P.; Cibor, J.; Debbe, R.; et al. Charged meson rapidity distributions in central Au+Au collisions at $\sqrt{s_{N N}}=$ $200 \mathrm{GeV}$. Phys. Rev. Lett. 2005, 94, 162301. [CrossRef] [PubMed]

26. Abbas, E.; Abelev, B.; Adam, J.; Adamova, D.; Adare, A.M.; Aggarwal, M.M.; Aglieri Rinella, G.; Agnello, M.; Agocs, A.G.; Agostinelli, A.; et al. Centrality dependence of the pseudorapidity density distribution for charged particles in $\mathrm{Pb}-\mathrm{Pb}$ collisions at $\sqrt{s_{N N}}=2.76$ TeV. Phys. Lett. B 2013, 726, 610-622. [CrossRef]

27. Adam, J.; Adamova, D.; Aggarwal, M.M.; Aglieri Rinella, G.; Agnello, M.; Agrawal, N.; Ahammed, Z.; Ahmad, S.; Ahn, S.U.; Aiola, S.; et al. Centrality dependence of the pseudorapidity density distribution for charged particles in $\mathrm{Pb}-\mathrm{Pb}$ collisions at $\sqrt{s_{N N}}=5.02 \mathrm{TeV}$. Phys. Lett. B 2017, 772, 567-577. [CrossRef]

28. Abelev, B.I.; Aggarwal, M.M.; Ahammed, Z.; Alakhverdyants, A.V.; Anderson, B.D.; Arkhipkin, D.; Averichev, G.S.; Balewski, J.; Barannikova, O.; Barnby, L.S.; et al. Long range rapidity correlations and jet production in high energy nuclear collisions. Phys. Rev. C 2009, 80, 064912. [CrossRef]

29. Chatrchyan, S.; Khachatryan, V.; Sirunyan, A.M.; Tumasyan, A.; Adam, W.; Bergauer, T.; Dragicevic, M.; Ero, J.; Fabjan, C.; Friedl, M.; et al. Long-range and short-range dihadron angular correlations in central $\mathrm{PbPb}$ collisions at $\sqrt{s_{N N}}=2.76 \mathrm{TeV}$. JHEP 2011, 7, 76. [CrossRef]

30. Adam, J.; Adamova, D.; Aggarwal, M.M.; Aglieri Rinella, G.; Agnello, M.; Agrawal, N.; Ahammed, Z.; Ahmad, S.; Ahn, S.U.; Aiola, S.; et al. Insight into particle production mechanisms via angular correlations of identified particles in pp collisions at $\sqrt{s}=7$ TeV. Eur. Phys. J. C 2017, 77, 569. [CrossRef]

31. Back, B.B.; Baker, M.D.; Ballintijn, M.; Barton, D.S.; Becker, B.; Betts, R.R.; Bickley, A.A.; Bindel, R.; Busza, W.; Carroll, A.; et al. Pseudorapidity Distribution of Charged Particles in d+Au Collisions at $\sqrt{s_{N N}}=200 \mathrm{GeV}$. Phys. Rev. Lett. 2004, 93, 082301. [CrossRef]

32. Acharya, S.; Acosta, F.-T.; Adamova, D.; Adhya, S.P.; Adler, A.; Adolfsson, J.; Aggarwal, M.M.; Aglieri Rinella, G.; Agnello, M.; Ahammed, Z.; et al. Charged-particle pseudorapidity density at mid-rapidity in p-Pb collisions at $\sqrt{s_{N N}}=8.16$ TeV. Eur. Phys. J. C 2019, 79, 307. [CrossRef]

33. Vechernin, V.V.; Ivanov, K.O.; Neverov, D.I. Two-particle correlation function and dihadron correlation approach. Phys. At. Nucl. 2016, 79, 798-806. [CrossRef]

34. Belokurova, S.N.; Vechernin, V.V. Calculation of long-range rapidity correlations in the model with string fusion on a transverse lattice. Phys. Part. Nucl. 2020, 51, 319-322. [CrossRef]

35. Adam, J.; Adamova, D.; Aggarwal, M.M.; Aglieri Rinella, G.; Agnello, M.; Agrawal, N.; Ahammed, Z.; Ahmed, S.; Ahn, S.U.; Aimo, I.; et al. Forward-backward multiplicity correlations in pp collisions at $\sqrt{s}=0.9$, 2.76 and 7 TeV. JHEP 2015, 5, 097. [CrossRef] 
36. Braun, M.A.; Pajares, C. Elliptic flow from colour strings. Eur. Phys. J. C 2011, 71, 1558. [CrossRef]

37. Kovalenko, V.N. Modelling of exclusive parton distributions and long-range rapidity correlations for $\mathrm{pp}$ collisions at the LHC energy. Phys. Atom. Nucl. 2013, 76, 1189-1195. [CrossRef]

38. Kovalenko, V.; Vechernin, V. Model of pp and AA collisions for the description of long-range correlations. Proc. Sci. 2012, Baldin-ISHEPP-XXI, 077.

39. Braun, M.A.; Pajares, C.; Vechernin, V.V. Anisotropic flows from colour strings: Monte-Carlo simulations. Nucl. Phys. A 2013, 906, 14-27. [CrossRef]

40. Braun, M.A.; Pajares, C.; Vechernin, V.V. Ridge from strings. Eur. Phys. J. A 2015, 51, 44. [CrossRef]

41. Kovner, A.; Lublinsky, M. Angular correlations and high energy evolution. Phys. Rev. D 2011, 83, 034017. [CrossRef]

(C) 2020 by the authors. Licensee MDPI, Basel, Switzerland. This article is an open access article distributed under the terms and conditions of the Creative Commons Attribution (CC BY) license (http:/ / creativecommons.org/licenses/by/4.0/). 